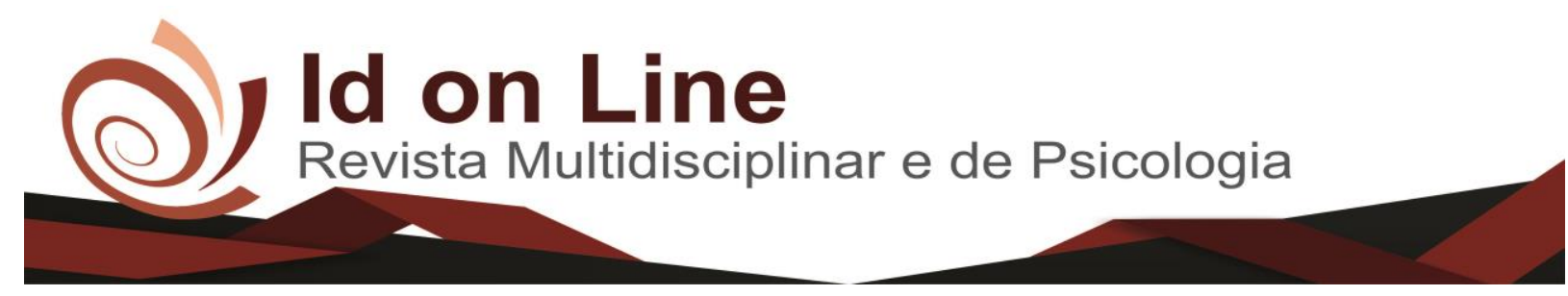

Artigo

\title{
Função Sexual e Qualidade de Vida de Mulheres Submetidas à Histerectomia
}

Jéssika Lange Castro dos Santos ${ }^{1}$; Rosana Porto Cirqueira ${ }^{2}$; Luciana Santos de Albuquerque ${ }^{3 ;}$ Thaís Dutra Rodrigues ${ }^{4}$ Juliana Barros Ferreira ${ }^{5}$

\begin{abstract}
Resumo: Este estudo tem como objetivo analisar a qualidade de vida e a função sexual de mulheres histerectomizadas. Foi realizado um estudo do tipo observacional, transversal e analítico. Utilizou-se como instrumento de coleta de dados um questionário sociodemográfico, além do FSFI que avaliou a função sexual, e o questionário que avalia a qualidade de vida o WHOQOL- bref. A amostra foi composta por 28 mulheres histerectomizadas, casadas, com baixa renda, e idade média entre 40-50 anos. Verificou-se que a qualidade de vida geral foi regular e a maioria das participantes não apresentou disfunção sexual. Na correlação da qualidade de vida e função sexual, obtida através teste U Mann-Whitney, os domínios avaliados não apresentaram significância $(\mathrm{p}<0,005)$. Estudos com um acompanhamento de tempo maior são necessários para avaliar a QV e a função sexual destas mulheres submetidas à histerectomia
\end{abstract}

Palavras-Chave: Disfunção sexual fisiológica. Sexualidade. Histerectomia. Qualidade de vida. Mulheres

\section{Sexual Function and Quality of Life of Women Submitted to Hysterectomy}

\begin{abstract}
This study has as goal analyze the quality of life and the sexual function of the hysterectomized women. It was realized a study of the observational, transversal and analytical type. The sociodemographic questioner was used as the instrument of collecting of data, furthermore the FSFI that evaluated the sexual function, and the questioner that rate the quality of life, the WHOQOL-bref. The sample was composed by 28 hysterectomized women, married, with low income and average age around 40-50 years. Checked that the general quality of life was regular, and the majority of the participants did not show sexual disfunction. In the correlation between quality of life and sexual function, obtained by U Mann-Whitney test, the domains evaluated did not present significance $(\mathrm{p}<0.005)$. It is suggested the realization of new studies that approach the topic and looks for statistically establish this correlation.
\end{abstract}

Keywords: Physiological sexual dysfunction. Sexuality. Hysterectomy. Quality of life. Woman.

\section{Introdução}

${ }^{1}$ Graduanda em Fisioterapia pela Faculdade Independente do Nordeste - FAINOR E-mail: lange bjl@ hotmail.com; ${ }^{2}$ Fisioterapeuta . Docente da FTC e FAINOR . Mestranda em Saúde Coletiva- UFBA/Ba, Brasil

E-mail: porto_rosana@yahoo.com.br;

${ }^{3}$ Fisioterapeuta. Docente da FAINOR. Especialista em Pilates: Atenção Múltipla a Saúde- Faculdade Guanambi/BA; 4Graduanda em Fisioterapia pela Faculdade Independente do Nordeste - FAINOR.E-mail: tai.d.r@hotmail.com;

5Fisioterapeuta, Docente da FAINOR e FTC em Vitória da Conquista/BA. Mestre em Tecnologias em Saúde da EBMSP. Salvador - BA, Brasil. E-mail:: julibarros78@hotmail.com. 
O termo histerectomia é empregado para designar a remoção do útero por acesso cirúrgico, e pode ser classificado como histerectomia total, subtotal e radical, usando uma das três principais abordagens: histerectomia abdominal $(\mathrm{AH})$, histerectomia vaginal $(\mathrm{VH})$ ou histerectomia laparoscópica (LH) (REAL 2012; PORTELA, 2015).

As patologias que acometem a pelve da mulher configuram um sério problema de saúde pública, que afetam órgãos que possuem valores simbólicos relacionados à feminilidade (SILVA; SANTOS; VARGENS, 2016). Dessa forma a remoção do útero pode ser realizada para intervir sobre diversas complicações ginecológicas, como por exemplo, miomas uterinos, sangramento vaginal anormal, prolapsos de órgãos, câncer cervical e endometriose (TEIXEIRA, BATISTA 2016).

No Brasil, tornou-se uma indicação cada vez mais comum, realizada em mulheres ainda em fase reprodutiva (REAL 2012). Segundo os dados do DATASUS, até junho de 2011, foram realizadas 27.867 cirurgias pelo Sistema Único de Saúde (SUS). Em 2014 foram realizadas cerca de 83 milhões histerectomias totais (LUNELLI, 2014; FREITAS, 2015).

Durante o procedimento cirúrgico da histerectomia, pode ocorrer lesão do plexo hipogástrico inferior bilateral. Este plexo é responsável pelas inervações simpáticas e parassimpáticas da região pélvica, especificamente no trajeto da ressecção do colo do útero (TOZO et al,.2011). A abordagem cirúrgica da histerectomia é capaz de provocar o encurtamento do canal vaginal, redução da libido e menor ocorrência de orgasmos pós penetração (FALEIROS, 2016). Freitas (2016), alerta para uma associação entre a histerectomia e conflitos psicológicos, os quais provocam mudanças importantes no comportamento, no desejo sexual e na qualidade de vida (QV).

O impacto da histerectomia na função sexual não está claro na literatura, e a prevalência de disfunção sexual feminina varia amplamente, principalmente devido às diferenças metodológicas da cirurgia (ILLIANO;GIANNITSAS; COSTANTINI, 2016). A compreensão das implicações e avaliação da qualidade de vida advindas desse procedimento é de extrema importância para que estas pacientes estejam preparadas para prováveis complicações que possam surgir (NAUTHON, 1997). A literatura aborda que avaliar a QV torna-se essencial, pois pode determinar novas técnicas de tratamento e analisar as respostas funcionais das diferentes intervenções clínicas sob a visão das pacientes (FREITAS 2015). 
Dessa forma, a finalidade deste trabalho é justificada pela baixa investigação acerca das repercussões que a histerectomia causa na sexualidade feminina, e seus possíveis impactos na QV. Entretanto, este estudo tem como objetivo analisar a QV e a função sexual de mulheres histerectomizadas, caracterizar o perfil sociodemográfico de mulheres submetidas à histerectomia e verificar a correlação entre a função sexual e a QV em mulheres histerectomizadas.

\section{Metodologia}

Foi realizado um estudo do tipo observacional com delineamento transversal, analítico e abordagem quantitativa, com 28 mulheres histerectomizadas em uma Unidade Básica de Saúde (UBS), localizada no município de Vitória da Conquista- Ba. A seleção da amostra foi não probabilística, por conveniência, tendo como critério de inclusão, mulheres com idade entre 18 a 70 anos com vida sexual ativa e que foram submetidas a uma histerectomia. Foram excluídas pacientes analfabetas, pacientes com déficit cognitivo e/ou neurológico, e limitações físicas que pudessem interferir na função sexual. Para coleta de dados utilizou-se três questionários: um com dados sociodemográfico, outro que avalia a QV, o WHOQOLbref, e outro para avaliar a função sexual, o FSFI.

No questionário sociodemográfico foi verificado os dados referentes à idade, estado civil, escolaridade, profissão e renda familiar. Para avaliar a QV das mulheres, foi utilizada a versão abreviada em português, validado para o Brasil, e indicado pela Organização Mundial da Saúde, o questionário WHOQOL-bref. Este questionário é composto por 26 questões, divididas em 4 domínios: físico, psicológico, relações sociais e meio ambiente e possui um escore que varia de 0 a 100, sendo 0 o pior estado de QV, e 100 o melhor resultado da QV. A resposta do WHOQOL-bref é dada em uma escala de Likert de 5 pontos, dessa forma, os escores entre 81 e 100 classificariam uma QV muito boa, escores entre 61 e 80 classificariam uma QV boa, escores entre 41 e 60 classificariam uma QV nem ruim nem boa, escores entre 21 e 40 classificariam uma QV ruim. E, para finalizar, escores entre 0 a 20 classificariam uma QV muito ruim (KLUTHCOVSKYI; KLUTHCOVSKYI, 2010, PASCHOA, ZANEI; WHITAKER, 2007). 
Para avaliar a função sexual, foi utilizado o Female Sexual Function Index (FSFI). Este questionário é validado para o Brasil, e contêm 19 questões que avaliam a função sexual nas últimas quatro semanas. As opções de respostas recebem pontuação entre 0 a 5 de forma crescente em relação à presença da função questionada. Apenas nas questões sobre dor a pontuação é definida de forma invertida. Se o escore de algum domínio for igual à zero, significa que não foi referida pela entrevistada relação sexual nas últimas quatro semanas. Ao final é apresentado um escore total, resultado da soma dos escores de cada domínio multiplicada por um fator de cada domínio no escore total. A análise foi realizada reunindo as respostas em seis domínios diferentes: Desejo itens 1 e 2 x (0,6); Excitação itens 3, 4, 5 e 6 x $(0,3)$; Lubrificação itens 7, 8, 9, e 10 x (0,3); Orgasmo itens 11, 12 e 13x (0,4); Satisfação itens 14,15 e 16 x (0,4); Dor itens 17, 18 e 19x (0,4).. O escore varia de 2 a 36 pontos, se ele for menor ou igual a 26 designa disfunção sexual. (HENTSCHEL, 2007; PECHORRO, 2009).

Todos os questionários foram respondidos individualmente, em uma sala reservada, para garantir e preservar a privacidade das participantes. A pesquisa ocorreu nos meses de agosto e setembro de 2017 , duas vezes na semana no período vespertino, horário em que ocorriam os atendimentos médicos e da enfermagem na Unidade Básica de Saúde.

Foi realizada uma análise descritiva das variáveis do estudo, mediante a distribuição das variáveis categóricas (porcentagem), sendo calculadas medidas de tendência central (desvio padrão) e as diferenças estatísticas entre proporções (média) utilizando o pacote estatístico SPSS (Statistical Package for Social Sciences) versão 22.0. A plotagem das tabelas foi feita por meio do software Microsoft Office Excel 2013 Plus. Para a análise da comparação foi utilizado o teste U de Mann Whitney

A pesquisa foi aprovada pelo comitê de ética e pesquisa da FAINOR (CEP/FAINOR), conforme CAAE: 69862517.9.0000.5578 e parecer 2.132.352. Após esclarecimento do estudo obteve-se consentimento para inicio da coleta através da assinatura do Termo de Consentimento Livre e Esclarecido (TCLE) conforme preceitos éticos do Conselho Nacional de Saúde que constam na resolução 466/12. 


\section{Resultados}

Foram entrevistadas 29 pacientes histerectomizadas, dentre estas 1 foi excluída por não atender aos critérios de inclusão, ao final 28 entrevistas constituíram o resultado da amostra, os quais demonstraram que em sua maioria $57,1 \%$ das mulheres possuíam idade entre 40 e 50 anos, $75,0 \%$ são casadas, $46,4 \%$ possuem o ensino médio completo e $42,9 \%$ estão empregadas, com renda até 2 salários mínimos 42,9\% como descreve a tabela 1.

Tabela 1. Características sociodemográficas da amostra. Vitória da Conquista - BA, 2017.

\begin{tabular}{|c|c|c|c|}
\hline Variáveis & $\%$ respostas & $\mathbf{n}$ & $\%$ \\
\hline Faixa etária & 100 & & \\
\hline De 40 a 50 anos & & 16 & 57,1 \\
\hline De 50 a 60 anos & & 11 & 39,3 \\
\hline De 60 a 70 anos & & 1 & 3,6 \\
\hline Estado civil & 100 & & \\
\hline Casado & & 21 & 75,0 \\
\hline Solteiro & & 3 & 10,7 \\
\hline Divorciado & & 3 & 10,7 \\
\hline União estável & & 1 & 3,6 \\
\hline Escolaridade & 100 & & \\
\hline Ensino médio & & 13 & 46,4 \\
\hline Ensino fundamental & & 9 & 32,1 \\
\hline Pós-graduação & & 4 & 14,3 \\
\hline Analfabeta funcional & & 1 & 3,6 \\
\hline Ensino superior & & 1 & 3,6 \\
\hline Situação de emprego & 100 & & \\
\hline Empregada & & 12 & 42,9 \\
\hline Desempregada & & 9 & 32,1 \\
\hline Autônoma & & 7 & 25,0 \\
\hline Renda & 100 & & \\
\hline Até 2 salários mínimos & & 12 & 42,9 \\
\hline De 2 a 5 salários mínimos & & 6 & 21,4 \\
\hline Sem redimento/não sabe & & 6 & 21,4 \\
\hline De 5 a 10 salários mínimos & & 4 & 14,3 \\
\hline
\end{tabular}

n: número de participantes; \%: porcentagem

Fonte: Dados da pesquisa

Para avaliar a função sexual foi utilizado o questionário FSFI. Os dados obtidos nesse questionário contemplam os seguintes domínios: Desejo, excitação, lubrificação, orgasmo, satisfação e dor. 
De acordo com a Tabela 2, 67,9\% $(\mathrm{n}=19)$ das mulheres não apresentaram disfunção sexual, o comprometimento na função sexual foi relatado em 32,1\% ( $n=9)$ apresentando alteração em todos os domínios do instrumento. A menor média/desvio padrão foi referente ao domínio desejo $2,93 \%( \pm 0,92)$ e a maior média foi referente ao domínio dor $3,77 \%( \pm$ 1,56). De acordo com o FSFI resultados abaixo de 4,58\% comprovam disfunção sexual.

Tabela 2. Função sexual da amostra. Vitória da Conquista - BA, 2017.

\begin{tabular}{|c|c|c|c|c|}
\hline \multicolumn{2}{|r|}{ Variáveis } & $\begin{array}{c}\% \\
\text { Respostas }\end{array}$ & $\mathbf{n}$ & $\%$ \\
\hline FSFI $^{1}$ & & 7100 & & \\
\hline & Disfunção sexual & & 9 & 32,1 \\
\hline & Ausência de disfunção sexual & & 19 & 67,9 \\
\hline
\end{tabular}

${ }^{1}$ Female Sexual Function Index

Fonte: Dados da pesquisa.

A QV de mulheres submetidas à histerectomia foi avaliada com base no instrumento WHOQOL-bref, foi obtida pela média dos domínios físico, psicológico, relações sociais e meio ambiente, conforme tabela 3.

Em relação às facetas analisadas, o domínio psicológico foi o que obteve o maior escore, classificado como bom 71,4\%, o domínio físico foi o que obteve menor escore 50,0\%, classificado como regular. Desta forma, no que determina QV geral, apresentou-se regular obtendo uma média de 3,96\% $( \pm 0,96)$, classificação que também se aplica ao domínio da satisfação com a saúde com média de 3,57\% ( $\$ 95)$.

Tabela 3. Qualidade vida WHOQOL-bref. Vitória da Conquista - BA, 2017.

\begin{tabular}{lccc}
\hline Variáveis & Média & DP1 \\
\hline Qualidade de vida geral & & 3,96 & 0,96 \\
$\quad$ Percepção da qualidade de vida & \% respostas & $\mathrm{n}$ & 0,95 \\
$\quad$ Satisfação com a saúde & 100 & & $\%$ \\
Domínios WhoQol-bref & & 14 & 50,0 \\
Domínio físico & & 9 & 32,1 \\
$\quad$ Regular & 100 & 5 & 17,9 \\
$\quad$ Boa & & & \\
Necessita melhorar & & 20 & 71,4 \\
Domínio pisciológico & & 5 & 17,9 \\
$\quad$ Boa & & 3 & 10,7 \\
$\quad$ Regular & &
\end{tabular}


Domínio relações sociais

Boa

Regular

Necessita melhorar

Meio ambiente

Regular

Necessita melhorar

Boa
100

18

64,3

$8 \quad 28,6$

27,1

100

${ }^{1}$ Desvio Padrão

Fonte: Dados da pesquisa.

$\mathrm{Na}$ tabela 4, a média geral mostrou-se satisfatória, visto que apenas 9 (nove) participantes demonstraram comprometimento da função sexual, a média foi de 20,14 $( \pm 7,02)$.

Foi utilizado o teste U Mann-Whitney, para correlacionar QV a função sexual e verificar se a QV dessas mulheres é afetada pela disfunção. O resultado abaixo mostra que os valores descritos em cada domínio não apresentaram correlação entre QV e função sexual, pois nenhum atingiu significância p-valor igual ou menor que 0,05 .

Tabela 4. Correlação entre função sexual e Qualidade de vida. Vitória da Conquista - BA, 2017.

\begin{tabular}{|c|c|c|c|}
\hline Domínios WhoQuol-bref & $\begin{array}{l}\text { Ausência de disfunção } \\
(\mathbf{n}=19)\end{array}$ & $\begin{array}{l}\text { Presença de disfunção } \\
(\mathbf{n}=9)\end{array}$ & \multirow[t]{2}{*}{ p-valor } \\
\hline & Média \pm DP1 & Média \pm DP & \\
\hline $\begin{array}{l}\text { Percepção da } \\
\text { qualidade de vida }\end{array}$ & $4,05 \pm 0,97$ & $3,77 \pm 1,01$ & 0,604 \\
\hline Satisfação com a saúde & $3,52 \pm 0,90$ & $3,66 \pm 0,86$ & 0,114 \\
\hline Domínio físico & $3,56 \pm 0,77$ & $3,76 \pm 0,59$ & 0,387 \\
\hline $\begin{array}{l}\text { Domínio psicológico } \\
\text { Domínio relações }\end{array}$ & $4,03 \pm 0,56$ & $3,83 \pm 0,57$ & 0,159 \\
\hline sociais & $3,92 \pm 0,69$ & $3,81 \pm 0,53$ & 0,379 \\
\hline Meio ambiente & $3,52 \pm 0,48$ & $3,28 \pm 0,80$ & 0,628 \\
\hline
\end{tabular}

Fonte: Dados da Pesquisa

\section{Discussão}

Conforme pode ser observado, o estudo em questão demonstrou maior prevalência de mulheres submetidas à histerectomia com idade acima de 40 anos e casadas, corroborando 
com um estudo realizado por Teixeira e Batista (2016), com participantes de 40 a 65 anos de idade, casadas e pós-histerectomizadas. Em um estudo realizado, em Portugal, por Silva (2014) com uma amostra de 262 casos, a média de idade das mulheres que realizaram o procedimento foi de 48,9 anos. Por sua vez, Sbroggio; Giraldo e Gonçalves, (2008) desenvolveu um estudo com 164 pacientes que foram histerectomizadas há mais de 3 meses e menos de 5 anos, com idade entre 25 a 50 anos.

O estudo de Villar, Silva (2010) descreveu o perfil socioeconômico e cultural de 30 mulheres histerectomizadas. As participantes em sua maioria possuíam nível superior, estavam empregadas e com renda familiar que variou de $\mathrm{R} \$ 800,00$ a $\mathrm{R} \$ 15.000,00$. Divergindo dos resultados encontrados neste estudo, onde a renda foi de até dois salários mínimos.

Segundo Silva (2014) o nível socioeconômico e cultural tem associação entre a satisfação sexual e a remoção do útero. Homens e mulheres de classes econômicas distintas, longe do acesso ao mercado da beleza e livres dos incômodos extintos pela cirurgia, demonstram maior satisfação e prazer sexual. Já a mulher de classe média alta, associa o corpo à saúde estética, induzindo-a a perceber a histerectomia como responsável por seu desempenho sexual, pela visão de se sentir menos feminina. Diante disto os resultados mostram que fatores econômicos podem influenciar na percepção da autoimagem e induzir repercussões negativas no autoconceito de feminilidade. Por outro lado, estes mesmos fatores podem não produzir efeitos negativos, fazendo com que a histerectomia possa ser percebida de forma positiva pela melhora da saúde.

O presente estudo demonstrou que a QV geral, apresentou-se regular. Learman et al, (2002) afirma que os benefícios e desvantagens da remoção do útero foram amplamente investigados, porém, nenhum resultado mostrou diferença na QV. Já em uma revisão sistemática realizada por Kluivers et al. (2008), os dados mostram que a histerectomia laparoscópica é igual, ou melhor, em termos de saúde pós-operatória e QV. Além de mostrar que a vantagem de uma melhor QV deve ser compensada contra o aumento do risco de complicações. Os resultados de uma meta-análise de ensaios controlados randomizados realizada por Yi et al. (2011), mostram que a QV é o principal resultado para avaliar a abordagem da histerectomia. Para pacientes e cirurgiões, a histerectomia vaginal via laparoscópica é uma escolha melhor do que a histerectomia abdominal. Em um ensaio clínico randomizado e controlado em 59 participantes, que teve como objetivo investigar se 
há alteração da QV pós histerectomia laparoscópica ou via abdominal, através do questionário SF -36 foi comprovado que houve melhora da QV em pacientes submetidas à histerectomia por via laparoscópica, e as maiores pontuações foram encontradas nos domínios: aspecto físico, aspectos sociais e vitalidade (NIEBOER et al., 2012).

Em uma pesquisa realizada por Peña (2004), com mulheres mexicanas, foi possível visualizar que a angústia induzida pela perda de um órgão reprodutor, produziu mais reflexos negativos ao contexto social do que ao contexto biológico. Dessa forma, a compreensão sobre crenças e culturas do meio em que vivem, repercutiu sobre diversos aspectos socioculturais que possibilitaram perceber a histerectomia como coadjuvante de problemas relacionados à sua QV. O estudo de Silva, Santos e Vargens (2010), concluiu que as mulheres esboçaram boa aceitação após o procedimento de retirada do útero, em decorrência do alívio dos sintomas como dor e sangramento, muitas vezes intensos, e que comprometiam a QV. Segundo Faleiros (2016), no pré-operatório transtornos importantes como a ansiedade, e a falta de informação sobre a própria sexualidade podem comprometer a busca pelo prazer no relacionamento conjugal, o que por consequência pode produzir prejuízos importantes na QV.

Ao analisar a função sexual das 28 mulheres histerectomizadas com a aplicação do questionário FSFI, obteve-se uma resposta positiva e satisfatória na maioria das participantes.

Lunelli (2014) avaliou a função sexual em 39 mulheres histerectomizadas e realizou aplicação do questionário que avalia a função sexual o QS-F, no período pré e pós cirúrgico de 6 meses, os resultados mostram que duas participantes pioraram sua satisfação sexual de bom-excelente para regular-bom e outras duas melhoraram seu desempenho/satisfação de nulo-ruim para ruim-desfavorável, onde o escore total antes da histerectomia foi $66,8 \pm 18,5$ e depois foi $66,0 \pm 15,3$ (P < 0,52). Em outro estudo de Salimena e Souza (2010), a maioria das pacientes declarou melhora da qualidade sexual após o procedimento da histerectomia. Estes resultados apresentam semelhança com o presente estudo, ao mostrar que não há relação da histerectomia com a disfunção sexual. Acredita-se, portanto que o procedimento possa não ter influência no comprometimento da satisfação sexual e que a qualidade de vida pode melhorar após o procedimento.

Dados do estudo realizado por Kamisaruk et al. (2012), mulheres histerectomizadas podem ter prejuízos na função sexual. Já Illiano, Giannitsas e Costantini (2016), mostra que não há prejuízos na função sexual de mulheres que foram submetidas à histerectomia, concordando com o presente estudo. O estudo de Real (2012), em sua revisão integrativa 
realizada com 17 artigos, concluiu que mulheres submetidas à histerectomia podem apresentar alterações no autoconceito. Estas alterações podem desenvolver sintomas psicossomáticos, depressivos e dificuldade de relacionamento sexual e conjugal, devido a associações psicológicas feitas entre a procriação e sexualidade. Compreende-se, portanto, que fatores subjetivos são capazes de provocar sentimentos conflitivos ligados a fatores psicológicos e interferir em aspectos relacionados à QV e função sexual. Os resultados apresentados, neste estudo, não fazem correlação da histerectomia com a função sexual, no entanto, pode-se sugerir que a eliminação dos sintomas pode contribuir para uma melhora na relação conjugal e por consequência na qualidade sexual.

Em um estudo retrospectivo realizado por Fram, Saleh e Sumrei, (2012), no período de dois anos por meio de entrevistas, em um hospital universitário da Jordânia, que incluiu 124 pacientes pós histerectomia, demonstrou que $75 \%$ das participantes apresentaram um impacto positivo na sexualidade, o que corrobora com os resultados obtidos no presente estudo. No estudo de Faleiros et al (2011), realizado com 11 mulheres no pré e pós operatório de histerectomia foi avaliado, através do FSFI e do questionário de sexualidade feminina de McCoy (QSFMccoy), que houve melhora da qualidade sexual no pós operatório. Estes resultados divergem do presente estudo, visto que, não foi encontrado correlação da QV com a função sexual, os escores médios obtidos após a aplicação dos questionários não demonstraram agravos nos índices de satisfação sexual na maioria das mulheres entrevistadas. Pode-se inferir, portanto que a avaliação da QV pode variar sobre diversos aspectos, e apresentar resultados significativos na função sexual, uma vez que saúde sexual é um fator importante na QV, visto que o bem estar geral está associado a uma função sexual satisfatória.

Em um estudo de Correia et al (2016), com 375 pacientes não histerectomizadas, foi avaliada a QV e função sexual de mulheres praticantes de atividade física. Os resultados obtidos após a aplicação dos questionários FSFI e o questionário que avalia a QV (SF-36), foram compatíveis com disfunção sexual. E a maior prevalência desta disfunção foi em mulheres com idade menor que 40 anos. A autora conclui que aspectos como, não ser fumante, ter muito boa percepção do estado de saúde, realizar atividades de lazer, ter atividade sexual, ter companheiro/cônjuge e não ter comorbidades contribuíram para uma melhor avaliação da QV. Assim como a literatura refere, múltiplos fatores podem contribuir para o surgimento de queixas referentes à sexualidade após a histerectomia, principalmente na perspectiva do pré operatório (FALEIROS, 2012). Já o estudo de Faria (2012), que avaliou a 
QV e a função sexual de mulheres com e sem incontinência urinária, mostrou que a QV sofreu impacto negativo na função sexual, e com maior evidência naquelas que apresentaram incontinência urinária. Pode-se compreender que a disfunção sexual feminina pode se apresentar por vários motivos como, por exemplo, patologias do aparelho geniturinário. Dessa forma, a associação de fatores pode gerar prejuízos à função sexual. Pode-se inferir que duas ou mais patologias associadas ao sistema urinário podem ser responsáveis pela disfunção sexual.

Uma das limitações deste estudo foi não ter sido realizada uma análise qualitativa da QV e não ter questionado o tipo de histerectomia realizada.

\section{Conclusão}

A QV mostrou-se regular, porém sem disfunção sexual na maioria das mulheres histerectomizadas. Contudo não existiu associação entre a qualidade e vida e a função sexual nesta população estudada. Estudos com um acompanhamento de tempo maior são necessários para avaliar a QV e a função sexual destas mulheres submetidas à histerectomia, com o fim de evitar viés de confundimento. Estes resultados podem ajudar a compreender a saúde sexual feminina, e os possíveis comprometimentos advindos da histerectomia, bem como, conscientizar sobre a importância de buscar ajuda profissional para minimizar os efeitos prováveis do procedimento.

\section{Referências}

BRASIL. DATASUS - Departamento de informática do SUS. Ministério da Saúde, 2013. Sistemas de informações hospitalares SUS (SIH/SUS). Disponível em: <http://datasus.saude.gov.br>. Acesso em 28/09/2017.

CORREIA, L. S. et al. Função sexual e qualidade de vida de mulheres: um estudo observacional. Rev Port Med Geral Fam, Lauro de Freitas-BA, v.32, n.6, p.405, 2016.

FALEIROS, N. P. A sexualidade em mulheres submetidas à histerectomia total e subtotal. Rev. Bras. Ginecol. Obstet, São Paulo, vol.33, n.3, p. 151. 2011. 
FARIA, K. PEDROSA, L. Avaliação da qualidade de vida e função sexual de mulheres com e sem incontinência urinária. Rev. Eletr. Enf. Uberaba, v.14, n.2. p. 73. abr./jun. 2012.

FRAM, K. M.; SALEH, S. S.; SUMREIN, I. A. Sexuality after hysterectomy at the Hospital of the University of Jordan: a teaching hospital experience. Arch Gynecol Obstet. p.287, v.4, n.8, abr. 2013.

FREITAS, C. B.; GOMES, N. P.; CAMPOS, L. M. Complicações Pós-cirúrgicas da histerectomia: Revisão integrativa. Revista Enferm UFPI, Salvador, v.4, n.3, p.86. jul./set. 2015.

FREITAS, C. B. et al. Complicações Pós-Cirúrgicas da Histerectomia: Revisão Integrativa. Revista Baiana de Enfermagem, Salvador, v.30, n.2, p.1-11, abr.jun. 2016.

SBROGGIO, A. M. R. Auto percepção corpórea e sexual de mulheres submetidas à histerectomia. Grupo Editorial Moreira Jr, Campinas, p. 260, 2008.

HENTSCHEL, H. et al. Validation of the female sexual function index (FSFI) for portuguese language. Revista HCPA, v.27, n.1, p.10. 2007.

ILLIANO, E. GIANNITSAS, K.; COSTANTINI, E. Hysterectomy and Sexuality. First Published, Jun. 2016.

KLUIVERS, KB; et.al .Total laparoscopic hysterectomy versus total abdominal hysterectomy with bilateral salpingo-oophorectomy for endometrial carcinoma: a randomised controlled trial with 5-year follow-up.Gynecol Surg; v.8 n.4 p.427, 2011.

LEARMAN, L. A. et al. A randomized comparison of total or supracervical hysterectomy: surgical complications and clinical outcomes. Obstet Gynecol, v.102, p.453. 2003.

LUNELLI, B. P. O impacto da histerectomia abdominal no desempenho/ satisfação sexual. Acm Arq. Catarin. Med, Florianópolis, v. 43, n.1, p. 49-53, jan./ mar. 2014.

NAUGHTON, M. J.; MCBEE, W. L. Qualidade de vida relacionada à saúde póshisterectomia. Grupo Editorial Moreira Jr, v.40, p. 947. 1997.

PECHORRO, P. et al. Validação portuguesa do Índice de Funcionamento Sexual Feminino (FSFI). Laboratório Psicol, v.7, n.1, p.33-44, 2009.

PEÑA, M. T. C. Histerectomia decorrente de complicações do parto em um grupo de mulheres mexicanas: uma visão sociocultural. Universidade de São Paulo Ribeirão Preto, p. 114, dez. 2004. 
PORTELA, E.; SOBRAL, M..; SOTO, G. Problemas sexuais decorrentes da histerectomia. Clin Invest Ginecol Obstet, Espanha, v.42, n. 1, mar. 2015.

REAL, A. A. et al. Os efeitos da histerectomia sobre a sexualidade feminina. Revista Saúde, v.38, n.2, p. 123130, 2012.

SALIMENA, A. M.; SOUZA, I. Cotidiano Da Mulher Pos-Histerectomia à Luz do Pensamento de Heidegger. Revista Brasileira de Enfermagem, Brasilia, v.63, n.2. 2010.

SILVA, P. L. N. Perfil das mulheres histerectomizadas: uma revisão bibliográfica. EFDeportes.com. Revista Digital, Buenos Aires, v.19, p.191, abr. 2014.

SILVA, C. M. C.; SANTOS, I. M. M.; VARGENS, O. C. A Repercussão da histerectomia na vida de mulheres em idade reprodutiva. Esc. Anna Nery Rev. Enferm. p.76-82. 2010.

TEIXEIRA, MR. BATISTA, EC. Vivências Cotidianas da Mulher Histerectomizada: Narrativas e Contextos: Rev Enfermagem e Saúde Coletiva. v. 1, n. 2, p. 91, Faculdade São Paulo - FSP, 2016.

TOZO, I. M. et al. Avaliação Da Sexualidade em Mulheres Submetidas à Histerectomia para Tratamento do Leiomioma Uterino. Rev Bras Ginecol Obstet, v.31, n.10, p.503. 2009.

VILLAR, A. S. E.; SILVA, L. R. História de Vidas de Mulheres Submetidas à Histerectomia. Revista Ciência Cuidado em Saúde, v.9, n.3, p.479-486. jul./set. 2010.

YI, Y. X. et al. Laparoscopic-assisted vaginal hysterectomy vs abdominal hysterectomy for benign disease: a meta-analysis of randomized controlled trials. Eur J Obstet Gynecol Reprod Biol, n.1, v.18. p.159. jun. 2011.

\section{Como citar este artigo (Formato ABNT):}

SANTOS, Jéssila L.C. dos; CIRQUEIRA, Rosana P.; ALBUQUERQUE, Luciana S. de; RODRIGUES, Thaís D.; FERREIRA, Juliana B. Função Sexual e Qualidade de Vida de Mulheres Submetidas à Histerectomia. Id on Line Revista Multidisciplinar e de Psicologia, 2017, vol.12, n.39, p.179-191. ISSN: 1981-1179.

Recebido: 20.11 .2017

Aceito: 25.11 .2017 